\title{
The symmetric equilibria of symmetric voter participation games with complete information
}

\author{
Georg Nöldeke ${ }^{\mathrm{a}, *}$, Jorge Peña ${ }^{\mathrm{b}}$ \\ ${ }^{a}$ Faculty of Business and Economics, University of Basel, Switzerland \\ ${ }^{b}$ Department of Evolutionary Theory, Max Planck Institute for Evolutionary Biology, \\ Germany
}

\begin{abstract}
We characterize the symmetric Nash equilibria of the symmetric voter participation game with complete information introduced by Palfrey and Rosenthal (1983). Our results confirm their conjecture on the existence, multiplicity, and comparative statics of such equilibria and yield more precise information on how changes in team size affect the location of equilibria.

JEL classification: C72, D72.

Keywords: Costly voting, Participation games, Mixed strategy equilibrium, Polynomials in Bernstein form
\end{abstract}

${ }^{*}$ Corresponding author. Georg Nöldeke, Faculty of Business and Economics, University of Basel, Peter Merian-Weg 6, 4002 Basel, Switzerland. Tel: + 416126733 20. Fax: + 416126727 59. Email: georg.noeldeke@unibas.ch 


\section{Introduction}

In a seminal contribution to the theory of voter turnout 1 Rosenthal (1983) consider a participation game in which each voter decides whether to cast a vote for her favorite among two candidates or abstain. Participation costs and the benefit of the favored candidate winning the election, with the latter normalized to one, are the same for all voters and there is complete information about the numbers of voters favoring each of the two candidates. While a substantial part of the formal analysis and much of the discussion in Palfrey and Rosenthal (1983) considers the case in which both candidates have the same number of supporters and ties are broken with a coin toss, surprisingly little is known about the Nash equilibria of this symmetric voter participation game. In particular, the precise condition under which totally mixed symmetric equilibria exist, whether there can be more than two such equilibria, and their comparative statics in the size of the electorate are open questions. In this note we settle these questions.

Our first main result (Corollary 1) shows that for a given size of the electorate the number of totally mixed symmetric equilibria is either zero, one, or two, and provides the necessary and sufficient conditions for each of these cases, thereby verifying a corresponding conjecture in Palfrey and Rosenthal (1983, p. 29). Our second main result (Corollary 2) shows that for any participation cost in the interval $(0,1 / 2)$ the number of totally mixed symmetric equilibria is two if and only if the size of the electorate is above a critical threshold. Further, the voting probability in the low turnout equilibrium is strictly decreasing in the size of the electorate and converges to zero, whereas the voting probability in the high turnout equilibrium is strictly increasing in the size of the electorate and converges to one. The convergence properties of these equilibria are again in line with the conjecture in Palfrey and Rosenthal (1983, p. 29); the monotonicity results go beyond their conjecture.

There is no denying that the model we consider is a very stark and special one. However, understanding the structure of the equilibria in the model of Palfrey and Rosenthal (1983) is not only of interest from a historical perspective but provides information about more refined models of rational voting behavior. As explained in Kalandrakis (2007, 2009) the equilibria we consider here are robust to slight variations in the payoff structure of the

\footnotetext{
${ }^{1}$ See Dhillon and Peralta (2002) and Feddersen (2004) for surveys.
} 
game and survive in nearby games with incomplete information about players' participation costs or the number of players. Further, it is clear that any of the mixed strategy equilibria we study here also induces an equilibrium in a game in which the two teams supporting the two different candidates have different sizes: if these team sizes are, say, $s_{1}$ and $s_{2}$ with $s_{2}>s_{1}$, then assuming that $s_{2}-s_{1}$ voters of the second team abstain and all other voters play one of the mixed strategy equilibria for team size $s_{1}$ that we identify here, yields an equilibrium for the overall game. We also note that the correctness of the conjecture in Palfrey and Rosenthal (1983) is often taken for granted in discussions of their model, either implicitly (as in the surveys mentioned in Footnote 1) or explicitly (as in Demichelis and Dhillon (2010)).

In the following section we briefly describe the symmetric version of the voter participation game from Palfrey and Rosenthal (1983) and introduce our notation. Section 3 presents results. We conclude with a brief discussion. All proofs are in the Appendix.

\section{Model}

There are $2 s$ voters divided into two teams of equal size $s \geq 2$. Let $T_{1}=$ $\{1, \ldots, s\}$ be the members of the first team and $T_{2}=\{s+1, \ldots, 2 s\}$ be the members of the second team. There are two alternatives (candidates, policy proposals) denoted by $A_{1}$ and $A_{2}$. Members of team $T_{1}$ prefer alternative $A_{1}$ and members of $T_{2}$ prefer alternative $A_{2}$. All players $i=1, \ldots, 2 \mathrm{~s}$ simultaneously decide whether to vote in favor of their preferred alternative $\left(a_{i}=1\right)$ or to abstain $\left(a_{i}=0\right)$. The election is decided by simple majority rule with ties being broken by a fair coin toss. Players receive a benefit equal to 1 if their favored alternative wins and 0 otherwise. Voting entails a participation cost of $c \in(0,1 / 2)$. Players are risk neutral. Hence, for any player $i$ if $k$ other members of her own team and $\ell$ members of the other team vote and she chooses action $a_{i} \in\{0,1\}$, her payoff is

$$
\pi\left(k, \ell, a_{i}\right)= \begin{cases}1-a_{i} \cdot c & \text { if } k+a_{i}>\ell \\ 1 / 2-a_{i} \cdot c & \text { if } k+a_{i}=\ell \\ -a_{i} \cdot c & \text { if } k+a_{i}<\ell\end{cases}
$$

We focus on symmetric mixed strategy profiles in which all voters participate with probability $x \in[0,1]$. The assumption $c<1 / 2$ ensures that the strategy profile in which all agents participate $(x=1)$ is a symmetric Nash 
equilibrium, whereas the strategy profile in which all agents abstain $(x=0)$ is not $2^{2}$ In the following we consider the case of a totally mixed symmetric strategy profile in which all players vote with probability $x \in(0,1)$. As shown in Palfrey and Rosenthal (1983, eq. (13)) such a strategy profile is a Nash equilibrium if and only if

$$
2 c=P(x, s)
$$

where $P(x, s)$ is the pivot probability:

$$
\begin{aligned}
P(x, s)= & \sum_{k=0}^{s-1}\left(\begin{array}{c}
s-1 \\
k
\end{array}\right)\left(\begin{array}{l}
s \\
k
\end{array}\right) x^{2 k}(1-x)^{2 s-2 k-1} \\
& +\sum_{k=0}^{s-1}\left(\begin{array}{c}
s-1 \\
k
\end{array}\right)\left(\begin{array}{c}
s \\
k+1
\end{array}\right) x^{2 k+1}(1-x)^{2 s-2 k-2} .
\end{aligned}
$$

The logic behind the equilibrium conditions (1) - (2) is familiar. For any player $i$, (2) gives the probability that player $i$ casts the decisive vote when each of the other players votes with probability $x$ : The first sum covers the cases in which $k$ members of the opposing team as well as $k$ other members of player $i$ 's own team vote, so that if $i$ participates, her votes resolves a tie and her favored alternative will be selected with probability 1 (rather than 1/2). The second sum covers the cases in which $k+1$ members of the opposing team as well as $k$ other members of $i$ 's own team vote, so that if $i$ participates her vote induces a tie and her favored alternative will be selected with probability $1 / 2$ (rather than 0 ). Because player $i$ gains an expected benefit of $1 / 2$ whenever her vote is pivotal (and a benefit of zero when it is not), the indifference condition for a Nash equilibrium in which $i$ 's mixed strategy assigns strictly positive probability to both pure strategies is that her probability of casting the decisive vote is twice the cost of participation. This yields (1) as the necessary and sufficient condition for the strategy profile in which all voters participate with probability $x \in(0,1)$ to be a symmetric Nash equilibrium.

\footnotetext{
${ }^{2}$ It is easy to see that for $c>1 / 2$ the only Nash equilibrium (either in pure or mixed strategies) is the symmetric one in which no agent participates. In the knife-edge case $c=1 / 2$ there is one additional symmetric Nash equilibrium in which all agents participate (and also a multitude of asymmetric equilibria).
} 


\section{Results}

\subsection{Preliminaries}

We begin by observing that the pivot probability $P(x, s)$ can be rewritten in a way that is more conducive to further analysis. For this purpose it will be convenient to define

$$
\phi(k, \ell, s)=\frac{\left(\begin{array}{c}
s-1 \\
k
\end{array}\right)\left(\begin{array}{l}
s \\
\ell
\end{array}\right)}{\left(\begin{array}{c}
2 s-1 \\
k+\ell
\end{array}\right)}, k=0, \ldots, s-1, \ell=0, \ldots, s .
$$

The expression in (3) is the probability (as determined by the hypergeometric distribution) of $k$ successes in $s-1$ draws, without replacement, from a population of size $2 s-1$ containing $k+\ell$ successes. Hence, given that the total number of voters among all players but $i$ is $k+\ell, \phi(k, \ell, s)$ is the probability that of these voters exactly $k$ are in $i$ 's team and exactly $\ell$ are in the other team. Letting

$$
p(j, s)= \begin{cases}\phi(j / 2, j / 2, s) & \text { if } j=0,2, \ldots 2 s-2 \\ \phi((j-1) / 2,(j+1) / 2, s) & \text { if } j=1,3, \ldots 2 s-1\end{cases}
$$

then gives the probability that player $i$ is pivotal conditional on there being exactly $j$ voters among all the other players - indeed, if $j$ is even, then player $i$ is pivotal if and only if the same number of other players vote in both teams and if $j$ is odd she is pivotal if and only if there is one less voter among the other players in her team than there are voters in the other team. Throughout the following we will refer to $p(j, s)$ as the conditional pivot probability.

With the above definitions a simple manipulation of the two sums appearing in equation (2) yields the following result.

Lemma 1. For all $x \in[0,1]$ and $n \geq 2$, the pivot probability is given by

$$
P(x, s)=\sum_{j=0}^{2 s-1}\left(\begin{array}{c}
2 s-1 \\
j
\end{array}\right) x^{j}(1-x)^{2 s-1-j} p(j, s) .
$$

Expression (5) has a simple interpretation. Fix some player $i$. Given that all other players independently choose to vote with probability $x$, the term $\left(\begin{array}{c}2 s-1 \\ j\end{array}\right) x^{j}(1-x)^{2 s-1-j}$ is nothing but the probability that there will be exactly $j$ voters among all the other players. Multiplying this with the conditional 
pivot probability $p(j, s)$ and summing over all $j$ gives the pivot probability $P(x, s)$.

The representation of the pivot probabilities obtained in Lemma 1 is useful because much can be said about the structure of the conditional pivot probabilities $p(j, s)$. The following lemma summarizes some key properties that are of relevance for our subsequent analysis. The proof uses well-known properties of the probability mass function of the hypergeometric distribution (cf. Johnson et al. 2005). Alternatively, the results can be verified by straightforward (but tedious) calculations using (3) and (4).

Lemma 2. For each $s \geq 2$ the conditional pivot probabilities satisfy $y^{3}$

$$
\begin{aligned}
p(0, s) & =1 \\
p(j, s) & =p(2 s-1-j, s), j=0,1, \ldots, 2 s-1 \\
p(2 k, s) & =p(2 k-1, s), \quad k=1, \ldots, s-1 \\
p(2 k, s) & >p(2 k+1, s), \quad k=0, \ldots,\lceil(s-1) / 2\rceil-1
\end{aligned}
$$

Equation (6) is obvious: if there are no voters among the other agents, then the player under consideration is surely pivotal. The equalities in (7) assert that the conditional pivot probabilities are symmetric in the sense that a player is equally likely to be pivotal when there are exactly $j$ voters or exactly $j$ abstainers among the other agents. In particular, we also have $p(2 s-1, s)=1$. The meaning of (8) is that the conditional pivot probability is unaffected when, starting from an even $j$, the number of voters among the other players is reduced by one. In contrast, (9) states that increasing an even number of voters among the other players by one strictly decreases the conditional pivot probability if the resulting odd numbers of voters is strictly smaller than the team size. Observe that it follows from the symmetry property in (7) that the inequality in $(9)$ is reversed when $2 k+1>s$ holds.

Figure 1 illustrates the shape properties of the conditional pivot probabilities $p(j, s)$ established in Lemma 2 and also shows the corresponding pivot probability $P(x, s)$ for the case $s=8$. The close resemblance between the two functions illustrated in Figure 1 suggests that shape properties of $P(x, s)$

\footnotetext{
${ }^{3}$ In $(9)$ the expression $\lceil(s-1) / 2\rceil$ denotes the ceiling of $(s-1) / 2$, that is, the smallest integer greater or equal than $(s-1) / 2$. Consequently, $\lceil(s-1) / 2\rceil-1$ is the largest integer strictly smaller than $(s-1) / 2$.
} 


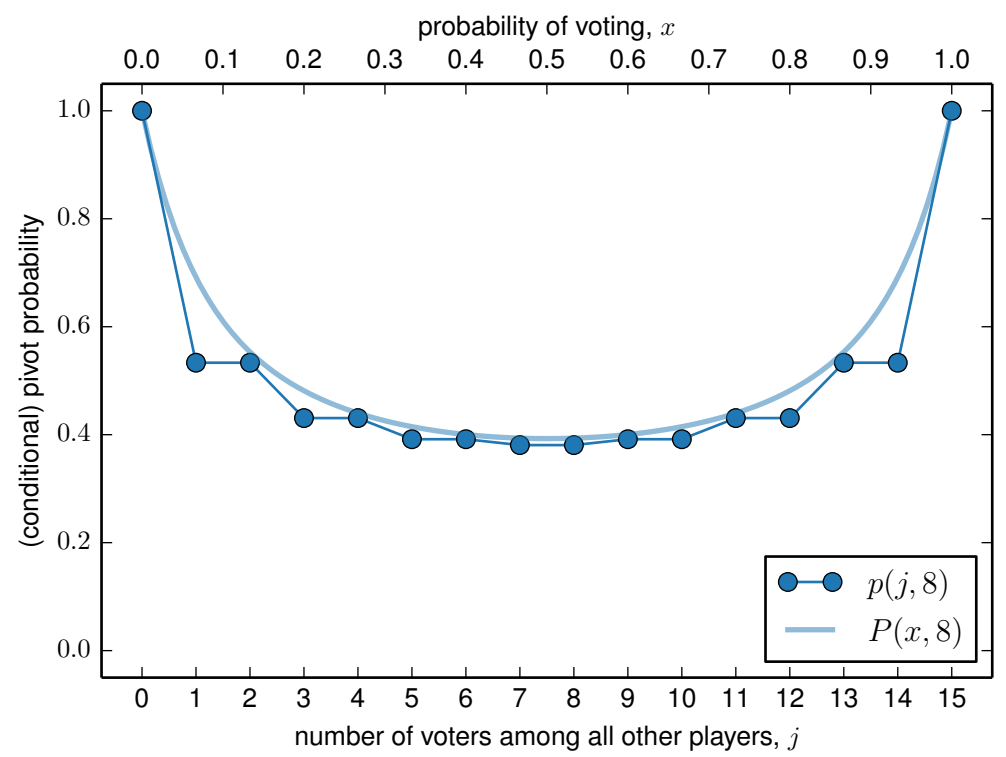

Figure 1: Conditional pivot probabilities $p(j, s)$ and corresponding pivot probability $P(x, s)$ for $s=8$.

can be inferred from the shape properties of $p(j, s)$. The following subsection verifies this.

\subsection{Existence, number, and comparative statics with respect to participation cost}

As totally mixed symmetric equilibria are given by the solutions to the equation $2 c=P(x, s)$, their existence, number and comparative statics are determined by the shape of the pivot probability $P(x, s)$. Our main result in this subsection (Proposition 1) describes how the pivot probability depends on $x$ for any given team size $s$. As an immediate corollary, we obtain a precise characterization of the number of totally mixed symmetric equilibria (which can range from zero to two) and a comparative static result describing how the location of the equilibria varies with the participation cost.

The expression for the pivot probability $P(x, s)$ obtained in Lemma 1 is a polynomial in Bernstein form (cf. Farouki, 2012) of degree $2 s-1$ with coefficients given by the finite sequence of conditional pivot probabilities $p(j, s)$. To obtain the following proposition we make use of three properties of such polynomials: (1) they preserve symmetry, (2) their derivatives can be 
again written as polynomials in Bernstein form, and (3) they are variation diminishing.4 In particular, we apply the symmetry property to infer symmetry of the pivot probability $P(x, s)$ in $x$ from the symmetry of the conditional pivot probabilities established in Lemma 2, Using the derivative property we can express the derivative of $P(x, s)$ with respect to $x$ as a polynomial in Bernstein form with coefficients given by a positive multiple of the difference between adjacent conditional pivot probabilities. Due to (8) and (9) in the statement of Lemma 2 we know that this finite sequence of coefficients has exactly one sign change (from negative to positive). An application of the variation diminishing property then implies that the derivative of $P(x, s)$ has exactly one root. By symmetry, this root is located at $x=1 / 2$. We thus obtain:

Proposition 1. For each $s \geq 2$, the pivot probability $P(x, s)$ satisfies $P(0, s)=$ $P(1, s)=1$ and is (1) symmetric in $x: P(x, s)=P(1-x, s)$ for all $x \in[0,1]$ and (2) strictly decreasing in $x$ in the interval $[0,1 / 2]$ and strictly increasing in $x$ in the interval $[1 / 2,1]$.

For $x \in(0,1)$ let

$$
c(x, s)=P(x, s) / 2 .
$$

denote the participation cost that solves (1) and let

$$
\underline{c}(s)=c(1 / 2, s)=\left(\frac{1}{2}\right)^{2 s-1}\left(\begin{array}{c}
2 s-1 \\
s
\end{array}\right)<\frac{1}{2},
$$

where the second equality is from Proposition 6 (a) in Palfrey and Rosenthal (1983) and the inequality is immediate from $P(1 / 2, s)<P(0, s)=P(1, s)=1$. It is a trivial implication of Proposition 1 that with participation cost $\underline{c}(s)$ the unique totally mixed symmetric equilibrium is given by $x=1 / 2$ and that $c(x, s)=c(1-x, s)>\underline{c}(s)$ holds for all $x \neq 1 / 2$ in $(0,1)$. Hence, we have proven the first two parts of the conjecture in Palfrey and Rosenthal (1983, p. 29). Observing that $P(0, s)=P(1, s)=1$ implies $c(0, s)=c(1, s)=$ $1 / 2$, we may reformulate these observations in terms of the following result,

\footnotetext{
${ }^{4}$ Roughly speaking, a transformation is variation diminishing if it reduces the number of sign changes (from positive to negative or vice versa). See Karlin (1968) for precise definitions and Brown et al. (1981) for a gentle introduction in a statistical context. For previous applications in economics, see Jewitt (1987) and Chakraborty (1999).
} 
characterizing the number and location of totally mixed symmetric equilibria in terms of the participation costs.

Corollary 1. For each $s \geq 2$ the following holds:

1. If $c<\underline{c}(s)$, there exists no totally mixed symmetric Nash equilibrium.

2. If $c=\underline{c}(s), x=1 / 2$ is the unique totally mixed symmetric Nash equilibrium.

3. If $c \in(\underline{c}(s), 1 / 2)$, there exist exactly two totally mixed symmetric Nash equilibria $x_{\mathrm{L}}(c, s)$ and $x_{\mathrm{H}}(c, s)$, satisfying $0<x_{\mathrm{L}}(c, s)<1 / 2<$ $x_{\mathrm{H}}(c, s)<1$. Further, $x_{\mathrm{L}}(c, s)+x_{\mathrm{H}}(c, s)=1$ holds for all $c$ in the indicated interval and $x_{\mathrm{L}}(c, s)$ is strictly decreasing in $c$ with $\lim _{c \rightarrow \underline{c}(s)} x_{\mathrm{L}}(c, s)=$ $1 / 2$ and $\lim _{c \rightarrow 1 / 2} x_{\mathrm{L}}(c, s)=0$.

Because $\underline{c}(s)<1 / 2$ holds, the range of cost values such that both the low participation equilibrium $x_{\mathrm{L}}(c, s)$ and the high participation equilibrium $x_{\mathrm{H}}(c, s)$ exist is non-empty for all team sizes $s \geq 2$. Further, as $\lim _{s \rightarrow \infty} \underline{c}(s)=$ 0 holds (cf. Palfrey and Rosenthal, 1983, Proposition 6 (c)), it follows from Corollary 1 . 3 that for all $c \in(0,1 / 2)$ there exists $S$ such that for all $s \geq S$ both the low participation equilibrium $x_{\mathrm{L}}(c, s)$ and the high participation equilibrium $x_{\mathrm{H}}(c, s)$ (and no other totally mixed symmetric Nash equilbria) exist. While this observation closely resembles the third part of the conjecture in Palfrey and Rosenthal (1985), these authors claim more: namely, that for any given cost level $c \in(0,1 / 2)$ the equilibria $x_{\mathrm{L}}(c, s)$ and $x_{\mathrm{H}}(c, s)$ not only exist for $s$ sufficiently large but also satisfy $\lim _{s \rightarrow \infty} x_{\mathrm{L}}(c, s)=0$ and $\lim _{s \rightarrow \infty} x_{\mathrm{H}}(c, s)=1$. In the following subsection we confirm this last part of the conjecture and, in addition, address the more general question of how a change in team size affects the location of equilibria.

\subsection{Team-size effect}

We proceed as in the previous subsection by first exploiting Lemmas 1 and 2 to determine how the pivot probability $P(x, s)$ depends on team size $s$ (Propositions 2 and 3 ) and then exploiting these results to determine the team-size effect, that is, how the location of totally mixed symmetric equilibria depends on the size of the teams.

The results we obtain for the pivot probability are very intuitive: given any symmetric strategy profile in which all players mix with probability 


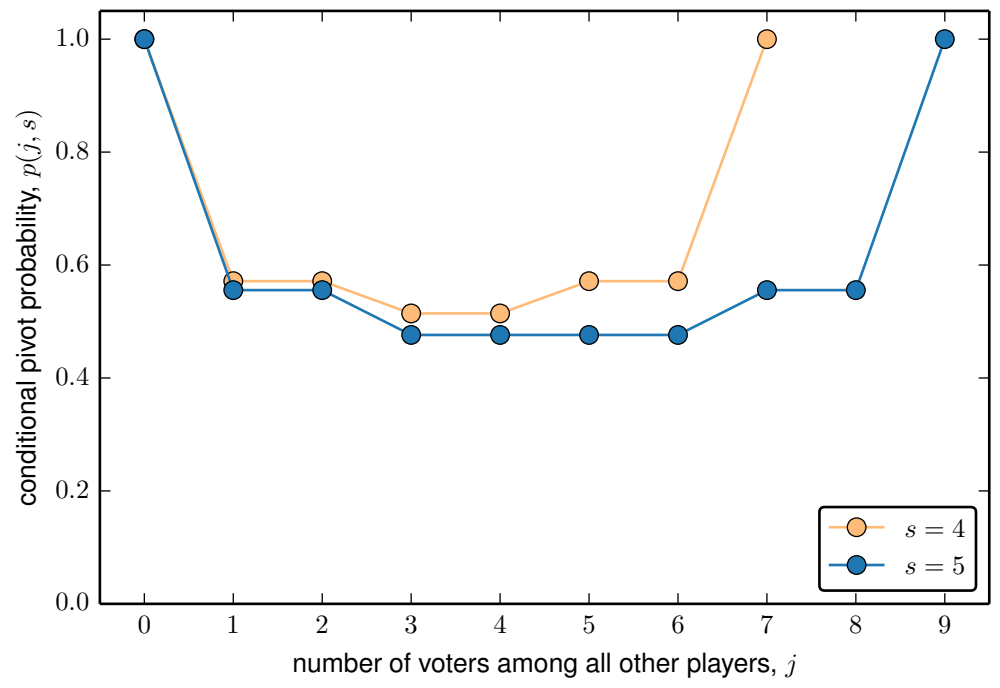

Figure 2: Conditional pivot probabilities $p(j, s)$ for $s=4$ and $s=5$. As established in Lemma 3. $p(j, s)>p(j, s+1)$ holds true for all $j=1, \ldots, 2 s-1$.

$x \in(0,1)$, a larger team size results in a lower pivot probability and this pivot probability converges to zero as team size goes to infinity. Establishing these results, however, is a non-trivial task and requires a number of steps. We begin with the observation that conditional pivot probabilities satisfy a monotonicity property.

Lemma 3. For each $s \geq 2$ the conditional pivot probabilities satisfy

$$
\begin{aligned}
p(j, s) & >p(j, s+1), j=1, \ldots, 2 s-1 \\
p(2 s-1-j, s) & >p(2 s+1-j, s+1) j=1, \ldots, 2 s-1
\end{aligned}
$$

We can offer a partial intuition for the result in Lemma 3. First, observe that by the symmetry of the conditional pivot probabilities (equation (7) in Lemma 22 the two statements in $(12)$ and $(13)$ are actually equivalent. (We state both sets of inequalities as both will be used in subsequent steps of the argument.) Hence, we may focus on the inequalities in (12) that we illustrate in Figure 2. Consider the extreme case $j=2 s-1$. In this case the conditional pivot probability is 1 when team size is $s$ because all other players vote, whereas it is strictly below one when team size is $s+1$ as there is the possibility that the two abstainers among the other agents happen to be in 
the same team. This implies $p(2 s-1, s)>p(2 s-1, s+1)$. At the other extreme, there is only one voter among the other players $(j=1)$. Because there is one less other player in the own team of any player $i$ than in the other team, the probability that the voter is in the other team, which is the case in which player $i$ is pivotal, exceeds one-half for any team size $s$. Indeed the relevant probability is simply given by $p(1, s)=s /(2 s-1)>1 / 2$, i. e. , the probability that a randomly sampled co-player is in the other team rather than in player $i$ 's team. This yields $p(1, s)>p(1, s+1)$. Considering these extreme cases identifies two distinct effects of an increase in team size on the conditional pivot probabilities. For $j$ between these extremes one would expect both of these effects to be at work and hence, as both effects tend to lower the conditional pivot probabilities when team size increases, the result in 12 .

The monotonicity of the conditional pivot probabilities established in Lemma 3 is suggestive but does not immediately imply a corresponding monotonicity property for the pivot probability $P(x, s)$. The difficulty is that for given $x$ the binomial distribution governing $j$ depends, of course, on team size. To establish the following proposition, we thus exploit a further result for polynomials in Bernstein form, namely the degree elevation formula (Farouki, 2012 p. 391$)$. This allows us to write $P(x, s)$ as a polynomial of degree $2 s+1$ featuring the same binomial probabilities as those appearing in $P(x, s+1)$ and coefficients $p^{*}(j, s+1)$ that are convex combinations of the conditional pivot probabilities $p(j, s)$. Lemmas 2 and 3 imply that for $j=1, \ldots, 2 s$ these transformed coefficients are strictly lower than the conditional pivot probabilities $p(j, s+1)$ (see Figure 3 for an illustration). Since decreasing the coefficients of a polynomial in Bernstein form with given degree clearly decreases the polynomial, we thus obtain:

Proposition 2. For every $x \in(0,1)$ the pivot probability $P(x, s)$ is strictly decreasing in team size $s$.

Having established that pivot probabilities are strictly decreasing in team size for any totally mixed symmetric strategy profile, we turn to establish that these pivot probabilities converge to zero. The argument here is fairly standard. It proceeds by (i) showing that the probability that the ratio $j /(2 s-1)$ lies outside a $\delta$-neighbourhood of $x$ converges to zero as team size goes to infinity and (ii) providing an upper bound for the conditional pivot probabilities when the ratio $j /(2 s-1)$ lies in the neighbourhood that also converges to zero as team size goes to infinity. 


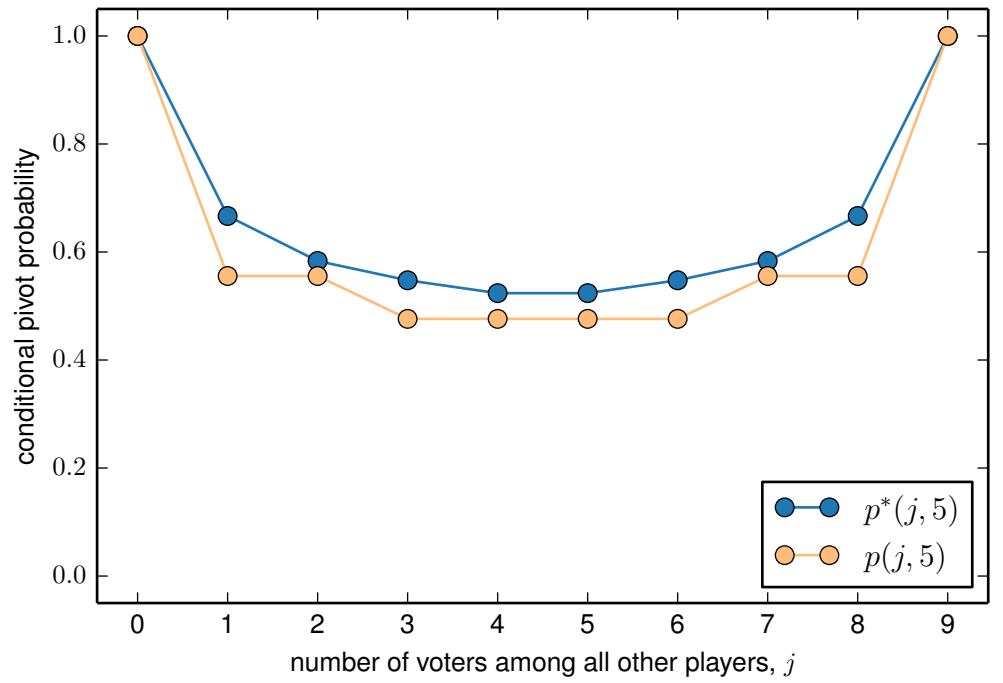

Figure 3: Conditional pivot probabilities $p(j, s+1)$ and degree-elevated conditional pivot probabilities $p^{*}(j, s+1)$ for $s=4$.

Proposition 3. For every $x \in(0,1)$ the pivot probability $P(x, s)$ satisfies $\lim _{s \rightarrow \infty} P(x, s)=0$.

Consider now any $c \in(0,1 / 2)$ and recall the definitions of the critical cost levels $c(x, s)$ and $c(s)$ from equations (10) and (11). From Propositions 2 and 3 we know that $\underline{c}(s)$ is strictly decreasing in $s$ with $\lim _{s \rightarrow \infty} \underline{c}(s)=0 \underbrace{5}$ It is then an immediate implication of Corollary 1 that there exists a critical team size $S$ such that two totally mixed symmetric equilibria exist if and only if team size is at least $S$. Further, provided that $s \geq S$ holds, the low participation equilibrium $x_{\mathrm{L}}(c, s)$ is given by the unique solution of the equation $c=c(x, s)$ in the interval $(0,1 / 2)$ and the high participation equilibrium is given by the unique solution of the same equation in the interval $(1 / 2,1)$. Combining the monotonicity properties of the pivot probabilities $P(x, s)$ established in Proposition 1 and 2 it is then immediate that an increase in team size causes both the low participation and the high participation equilibrium to move away from $1 / 2$, so that $x_{\mathrm{L}}(c, s)$ is a strictly decreasing and $x_{\mathrm{H}}(c, s)$ is a strictly increasing function of team size. It then follows from Proposition 3 that

\footnotetext{
${ }^{5}$ As noted after the statement of Corollary 1 the result $\lim _{s \rightarrow \infty} \underline{c}(s)=0$ has already been established in Palfrey and Rosenthal (1983).
} 
$x_{\mathrm{L}}(c, s)$ converges to 0 and $x_{\mathrm{H}}(c, s)$ converges to 1 as team size converges to infinity. We have thus established:

Corollary 2. For every $c \in(0,1 / 2)$ there exists a team size $S$ such that $c>$ $\underline{c}(s)$ holds if and only if $s \geq S$, so that the two totally mixed symmetric Nash equilibria $x_{\mathrm{L}}(c, s)$ and $x_{\mathrm{H}}(c, s)$ described in Corollary 1 . 3 exist if and only if $s \geq$ S. Further, these equilibria satisfy $x_{\mathrm{L}}\left(c, s^{\prime}\right)<x_{\mathrm{L}}(c, s)<x_{\mathrm{H}}(c, s)<x_{\mathrm{H}}\left(c, s^{\prime}\right)$ for all team sizes $s^{\prime}>s \geq S, \lim _{s \rightarrow \infty} x_{\mathrm{L}}(c, s)=0$, and $\lim _{s \rightarrow \infty} x_{\mathrm{H}}(c, s)=1$.

As suggested in Palfrey and Rosenthal (1983, p. 31) we may interpret the last part of Corollary 2 as the statement that with large number of voters there are exactly two totally mixed symmetric strategy equilibria, one with essentially everyone voting and one with essentially no voting.

\section{Discussion}

We have characterized the symmetric Nash equilibria of the symmetric voter participation game with complete information introduced by Palfrey and Rosenthal (1983), confirming their conjecture about the existence, multiplicity, and comparative statics properties of totally mixed symmetric equilibria and providing additional comparative statics results. As mentioned in Palfrey and Rosenthal (1985, p. 33), the truth of their conjecture implies further results for the case in which the participation cost is lower than the critical value required for the existence of a totally mixed symmetric equilibrium. In particular, for this case our results imply the existence of exactly two equilibria in which all members of team $T_{1}$ vote with probability $0<x_{1}<1$ and all members of team $T_{2}$ vote with probability $x_{2}=1-x_{1}$.

The key insight underlying our analysis is that for any symmetric strategy profile the pivot probability (which determines voter's incentive to participate) can be represented as a polynomial in Bernstein form with coefficients given by conditional pivot probabilities. While the results we obtain here hinge

on the specific properties of the conditional pivot probabilities, the theory of polynomials in Bernstein form is of broader game-theoretic relevance. For instance, in Peña et al. (2014) we use the shape-preservation properties of polynomials in Bernstein form to determine the number and evolutionary stability of symmetric equilibria in a broad class of binary contribution games ${ }^{6}$

\footnotetext{
${ }^{6}$ In Peña et al. (2015) we extend these insights to the biologically relevant cases of interactions in spatially structured populations and hence between relatives.
} 
We are confident that further applications to the analysis of other symmetric, two-action games with many players can be developed.

\section{Appendix}

Proof of Lemma 1. Setting $j=2 k$ and using (4), we have

$$
\begin{aligned}
\sum_{k=0}^{s-1}\left(\begin{array}{c}
s-1 \\
k
\end{array}\right)\left(\begin{array}{l}
s \\
k
\end{array}\right) x^{2 k}(1-x)^{2 s-2 k-1} & \\
& =\sum_{j=0,2, \ldots, 2 s-2}\left(\begin{array}{c}
s-1 \\
j / 2
\end{array}\right)\left(\begin{array}{c}
s \\
j / 2
\end{array}\right) x^{j}(1-x)^{2 s-1-j} \\
& =\sum_{j=0,2, \ldots, 2 s-2}\left(\begin{array}{c}
2 s-1 \\
j
\end{array}\right) x^{j}(1-x)^{2 s-1-j} p(j, s)
\end{aligned}
$$

Similarly, setting $j=2 k+1$ and using (4), we have

$$
\begin{aligned}
& \sum_{k=0}^{s-1}\left(\begin{array}{c}
s-1 \\
k
\end{array}\right)\left(\begin{array}{c}
s \\
k+1
\end{array}\right) x^{2 k+1}(1-x)^{2 s-2 k-2} \\
&=\sum_{j=1,3, \ldots 2 s-1}\left(\begin{array}{c}
s-1 \\
(j-1) / 2
\end{array}\right)\left(\begin{array}{c}
s \\
(j+1) / 2
\end{array}\right) x^{j}(1-x)^{2 s-1-j} \\
&=\sum_{j=1,3, \ldots, 2 s-1}\left(\begin{array}{c}
2 s-1 \\
j
\end{array}\right) x^{j}(1-x)^{2 s-1-j} p(j, s) .
\end{aligned}
$$

Substituting the above identities into the definition of $P(x, s)$ in (2) yields (5).

Proof of Lemma 2. From (4) we have $p(0, s)=\phi(0,0, s)$ and from (3) we have $\phi(0,0, s)=1$, so that $p(0, s)=1$ holds for all $s \geq 2$, thus establishing identity (6).

For the remainder of the proof we refer to properties of the probability mass function of the hypergeometric distribution listed in Johnson et al. (2005, p. 265-266). Consequently, it is useful to observe that in their notation for the probability mass function of the hypergeometric distribution the definition of $\phi$ in (3) becomes

$$
\phi(k, \ell, s)=f(k \mid k+\ell, s-1, s)
$$


and the definition of $p$ in (4) becomes

$$
p(j, s)= \begin{cases}f(j / 2 \mid j, s-1, s) & \text { if } j=0,2, \ldots 2 s-2 \\ f((j-1) / 2 \mid j, s-1, s) & \text { if } j=1,3, \ldots 2 s-1 .\end{cases}
$$

Suppose $j$ is even. Then $2 s-j-1$ is odd, so that using (14) the equality in (7) becomes

$$
f(j / 2 \mid j, s-1, s)=f(s-1-j / 2 \mid 2 s-1-j, s-1, s),
$$

which is immediate from Johnson et al. (2005, eq. (6.56), second line). The argument when $j$ is odd is analogous.

Using (14) the equality in (8) becomes

$$
f(k \mid 2 k, s-1, s)=f(k-1 \mid 2 k-1, s-1, s) .
$$

To establish this, it suffices to note that from equation (6.54) in Johnson et al. (2005) we have

$$
f(k-1 \mid 2 k, s-1, s)=\frac{k}{k+1} f(k-1 \mid 2 k-1, s-1, s)
$$

and from equation (6.52) in Johnson et al. (2005) we have

$$
f(k \mid 2 k, s-1, s)=\frac{k+1}{k} f(k-1 \mid 2 k, s-1, s) .
$$

Using (14) the inequality appearing in (9) becomes

$$
f(k \mid 2 k, s-1, s)>f(k \mid 2 k+1, s-1, s) .
$$

From equation (6.54) in Johnson et al. (2005) this inequality is equivalent to

$$
\frac{(s-k)(2 k+1)}{(k+1)(2 s-1-2 k)}<1 \Leftrightarrow k<\frac{s-1}{2},
$$

which is the desired result.

Proof of Proposition 1. From (5) in Lemma 1 it is immediate that $P(0, s)=$ $p(0, s)$ and $P(1, s)=p(2 s-1, s)$ holds, so that $P(0, s)=P(1, s)=1$ follows from Lemma 2 . 
By the symmetry of the Bernstein basis polynomials (Farouki, 2012, p. 389) and the symmetry of the conditional pivot probabilities, that is (7) in Lemma 2. we have

$$
\left(\begin{array}{c}
2 s-1 \\
j
\end{array}\right) x^{j}(1-x)^{2 s-1-j} p(j, s)=\left(\begin{array}{c}
2 s-1 \\
2 s-1-j
\end{array}\right)(1-x)^{2 s-1-j} x^{j} p(2 s-1-j, s)
$$

for all $x \in[0$,$] and j=0, \ldots, 2 s-1$ and thus

$$
\sum_{j=0}^{2 s-1}\left(\begin{array}{c}
2 s-1 \\
j
\end{array}\right) x^{j}(1-x)^{2 s-1-j} p(j, s)=\sum_{j=0}^{2 s-1}\left(\begin{array}{c}
2 s-1 \\
j
\end{array}\right)(1-x)^{j} x^{2 s-1-j} p(j, s) .
$$

for all $x \in[0,1]$. From equation (5) in Lemma 1 this implies the symmetry property $P(x, s)=P(1-x, s)$ for all $x \in[0,1]$.

Let $P^{\prime}(x, s)$ denote the derivative of $P(x, s)$ with respect to $x$. From the derivative property of polynomials in Bernstein form (Farouki, 2012, p. 391) we have

$$
P^{\prime}(x, s)=\sum_{j=0}^{2 s-2}\left(\begin{array}{c}
2 s-2 \\
j
\end{array}\right) x^{j}(1-x)^{2 s-2-j}[(2 s-1) \Delta p(j, s)],
$$

where for $j=0,1 \ldots, 2 s-2$

$$
\Delta p(j, s)=p(j+1, s)-p(j, s) .
$$

From (8) in Lemma 2 the equality $\Delta p(j, s)=0$ holds whenever $j$ is odd. From (9) we have $\Delta p(j, s)<0$ whenever $j$ is even and $j<s-1$ holds, whereas (by symmetry) $\Delta p(j, s)>0$ holds whenever $j$ is even and $j>(s-1)$ holds. If $j=s-1$ is even, then $\Delta p(j, s)=0$ is implied by the symmetry of the conditional pivot probabilities. It follows that the finite sequence of the coefficients $\Delta p(j, s)$ has exactly one sign change (when zero coefficients are ignored). Consequently, the variation diminishing property of polynomials in Bernstein form (Farouki, 2012, p. 390) implies that the equation $P^{\prime}(x, s)=0$ has exactly one solution in the interval $(0,1)$. By the symmetry of $P(x, s)$ in $x$ this solution must occur at $x=1 / 2$. Finally, as $(15)$ implies $P^{\prime}(0, s)=$ $(2 s-2) \Delta p(0, s)<0$ we obtain $P^{\prime}(x, s)<0$ for all $x \in[0,1 / 2)$ and (by symmetry) $P^{\prime}(x, s)>0$ for all $x \in(1 / 2,1]$. 
Proof of Lemma 3. From (7) it suffices to prove (12). Further, from (8) it suffices to establish the inequalities in $(12)$ for odd $j$. Setting $j=2 k+1$ and using (3) and (4) we thus have to show

$$
\begin{aligned}
& \frac{\left(\begin{array}{c}
s-1 \\
k
\end{array}\right)\left(\begin{array}{c}
s \\
k+1
\end{array}\right)}{\left(\begin{array}{c}
2 s-1 \\
2 k+1
\end{array}\right)}>\frac{\left(\begin{array}{l}
s \\
k
\end{array}\right)\left(\begin{array}{l}
s+1 \\
k+1
\end{array}\right)}{\left(\begin{array}{l}
2 s+1 \\
2 k+1
\end{array}\right)} \\
\Leftrightarrow & \left(\begin{array}{c}
s-1 \\
k
\end{array}\right)\left(\begin{array}{c}
s \\
k+1
\end{array}\right)\left(\begin{array}{l}
2 s+1 \\
2 k+1
\end{array}\right)>\left(\begin{array}{l}
s \\
k
\end{array}\right)\left(\begin{array}{l}
s+1 \\
k+1
\end{array}\right)\left(\begin{array}{l}
2 s-1 \\
2 k+1
\end{array}\right)
\end{aligned}
$$

for $k=0,1, \ldots, s-1$. Using the factorial formula for the binomial coefficients and eliminating identical factorials this is

$$
\begin{aligned}
& \frac{(s-1) !(2 s+1) !}{(s-1-k) !(s-1-k) !(2 s-2 k) !}>\frac{(s+1) !(2 s-1) !}{(s-k) !(s-k) !(2 s-2 k-2) !} \\
\Leftrightarrow & (2 s+1) 2 s(s-k)(s-k)>(s+1) s(2 s-2 k)(2 s-2 k-1) \\
\Leftrightarrow & (2 s+1)(s-k)>(s+1)(2 s-2 k-1) \\
\Leftrightarrow & k+1>0,
\end{aligned}
$$

establishing the desired result.

Proof of Proposition 2. Define, for convenience, $p(-2, s)=p(-1, s)=1$ and $p(2 s+1, s)=p(2 s, s)=1$, so that $p(-2, s)=p(-1, s)=p(0, s)$ and $p(2 s+1, s)=p(2 s, s)=p(2 s-1, s)$ holds. Applying the degree elevation formula for polynomials in Bernstein form (Farouki, 2012, p. 391) twice we can write

$$
P(x, s)=\sum_{j=0}^{2 s+1}\left(\begin{array}{c}
2 s+1 \\
j
\end{array}\right) x^{j}(1-x)^{2 s+1-j} p^{*}(j, s+1),
$$

where the elevated coefficients $p^{*}(j, s+1)$ are determined from the formula in the last line of Farouki (2012, p. 391). In particular, for all $j=0,1, \ldots, s$ there exists $\lambda_{-2}>0, \lambda_{-1}>0$, and $\lambda_{0}>0$ such that $\lambda_{-2}+\lambda_{-1}+\lambda_{0}=1$ and

$$
p^{*}(j, s+1)=\lambda_{-2} p(j-2, s)+\lambda_{-1} p(j-1, s)+\lambda_{0} p(j, s)
$$

holds. Similarly, for all $j=0,1, \ldots s$ there exists $\mu_{0}>0, \mu_{1}>0$, and $\mu_{2}>0$ such that $\mu_{0}+\mu_{1}+\mu_{2}=1$ and

$$
p^{*}(2 s+1-j, s+1)=\mu_{0} p(2 s-1-j, s)+\mu_{1} p(2 s-j, s)+\mu_{2} p(2 s+1-j, s)
$$


holds. Lemma 2 implies that for all $j=0,1, \ldots, s$ we have

$$
\begin{array}{r}
p(j-2, s) \geq p(j-1, s) \geq p(j, s) \\
p(2 s-1+j, s) \geq p(2 s-j, s) \geq p(2 s-1-j, s) .
\end{array}
$$

Applying these inequalities on the right sides of $(17)$ and $(18)$ we obtain

$$
p^{*}(j, s+1) \geq p(j, s) \text { and } p^{*}(2 s+1-j, s+1) \geq p(2 s-1-j, s)
$$

for $j=0,1, \ldots, s$. Lemma 3 then implies

$$
p^{*}(j, s+1)>p(j, s+1) \text { and } p^{*}(2 s+1-j, s+1)>p(2 s+1-j, s+1)
$$

for $j=1, \ldots, s$. As we also have $p^{*}(0, s+1)=p(0, s+1)=1$ and $p^{*}(2 s+$ $1, s+1)=p(2 s+1, s+1)=1,19$ implies

$$
\sum_{j=0}^{2 s+1}\left(\begin{array}{c}
2 s+1 \\
j
\end{array}\right) x^{j}(1-x)^{2 s+1-j} p^{*}(j, s+1)>\sum_{j=0}^{2 s+1}\left(\begin{array}{c}
2 s+1 \\
j
\end{array}\right) x^{j}(1-x)^{2 s+1-j} p(j, s+1)
$$

for all $x \in(0,1)$. Using (16) and (5) from Lemma 1 this is equivalent to $P(x, s)>P(x, s+1)$ for all $x \in(0,1)$.

Proof of Proposition 3. Given any $x \in(0,1)$, there exists $\delta>0$ and $\epsilon>0$ such that $\epsilon<x-\delta<x+\delta<1-\epsilon$ holds. Fix such a $\delta$ and $\epsilon$. From Lemma 1 we can then write

$$
\begin{aligned}
P(x, s)= & \sum_{\left\{j: \frac{j}{2 s-1}-x \mid \geq \delta\right\}}\left(\begin{array}{c}
2 s-1 \\
j
\end{array}\right) x^{j}(1-x)^{2 s-1-j} p(j, s) \\
& +\sum_{\left\{j:\left|\frac{j}{2 s-1}-x\right|<\delta\right\}}\left(\begin{array}{c}
2 s-1 \\
j
\end{array}\right) x^{j}(1-x)^{2 s-1-j} p(j, s) .
\end{aligned}
$$

As $p(j, s) \leq 1$ holds for all $j$ and $s$, a standard bound for the binomial probability distribution (e.g. Chang and Sederberg, 1997, Theorem 25.5) implies that the first sum in the above expression is smaller than $1 /\left(4(2 s-1) \delta^{2}\right)$ for all $s \geq 2$. Letting $k_{s}$ denote the largest integer satisfying the inequality $2 k /(2 s-1) \leq \epsilon$, we further have from Lemma 2 that the second sum in the above expression is bounded above by $p\left(2 k_{s}, s\right)$. Therefore, to prove the proposition it suffices to show that $p\left(2 k_{s}, s\right)$ converges to zero as $s$ converges to infinity. 
It is clear that for $s \rightarrow \infty$ we also have $k_{s} \rightarrow \infty$ with $\lim _{s \rightarrow \infty} k_{s} / s=\epsilon \epsilon$ $(0,1)$. We may write

$$
p\left(2 k_{s}, s\right)=\frac{\left(\begin{array}{c}
s-1 \\
k_{s}
\end{array}\right)\left(\begin{array}{c}
s \\
k_{s}
\end{array}\right)}{\left(\begin{array}{c}
2 s-1 \\
2 k_{s}
\end{array}\right)}=\left(\begin{array}{c}
2 k_{s} \\
k_{s}
\end{array}\right) \frac{\prod_{i=1}^{k_{s}}(s+1-i) \prod_{i=1}^{k_{s}}(s-i)}{\prod_{i=1}^{2 k_{s}}(2 s-i)} .
$$

Multiplying and dividing the fraction in the above expression by $2 s$ it is easy to see that

$$
\frac{\prod_{i=1}^{k_{s}}(s+1-i) \prod_{i=1}^{k_{s}}(s-i)}{\prod_{i=1}^{2 k_{s}}(2 s-i)} \leq\left(\frac{1}{2}\right)^{2 k_{s}} \frac{s}{s-k_{s}}
$$

holds. Because $s /\left(s-k_{s}\right)$ converges to the finite limit $1 /(1-\epsilon)$, it then suffices to establish

$$
\lim _{k_{s} \rightarrow \infty}\left(\begin{array}{c}
2 k_{s} \\
k_{s}
\end{array}\right)\left(\frac{1}{2}\right)^{2 k_{s}}=0
$$

to obtain the desired result. But this is immediate from Stirling's approximation for binomial coefficients of the form $\left(\begin{array}{c}2 n \\ n\end{array}\right)$ for large $n$.

\section{Acknowledgement}

Financial support by the Swiss National Science Foundation (Grant PBLAP3-145860 to Jorge Peña) is gratefully acknowledged. Jean-Michel Benkert provided useful comments on an earlier draft.

\section{References}

Brown, L. D., I. M. Johnstone, And K. B. MacGibbon (1981): "Variation diminishing transformations: a direct approach to total positivity and its statistical applications," Journal of the American Statistical Association, $76,824-832$.

Chakraborty, I. (1999): "Bundling decisions for selling multiple objects," Economic Theory, 13, 723-733.

Chang, G. And T. W. Sederberg (1997): Over and over again, Washington, DC: The Mathematical Association of America.

Demichelis, S. And A. Dhillon (2010): "Learning in elections and voter turnout," Journal of Public Economic Theory, 12, 871-896. 
Dhillon, A. And S. Peralta (2002): "Economic theories of voter turnout," The Economic Journal, 112, pp. F332-F352.

FARouki, R. T. (2012): "The Bernstein polynomial basis: A centennial retrospective," Computer Aided Geometric Design, 29, 379-419.

FedDersen, T. J. (2004): "Rational choice theory and the paradox of not voting," Journal of Economic Perspectives, 18, 99-112.

JEWITT, I. (1987): "Risk aversion and the choice between risky prospects: the preservation of comparative statics results," The Review of Economic Studies, 54, 73-85.

Johnson, N. L., A. W. Kemp, And S. Kotz (2005): Univariate discrete distributions, vol. 444, John Wiley \& Sons, 3 ed.

KALANDRAKIS, T. (2007): "On participation games with complete information," International Journal of Game Theory, 35, 337-352.

- (2009): "Robust rational turnout," Economic Theory, 41, 317-343.

Karlin, S. (1968): Total positivity, vol. I, Stanford, CA: Stanford University Press.

Palfrey, T. R. And H. Rosenthal (1983): "A strategic calculus of voting," Public Choice, 41, 7-53.

- (1985): "Voter participation and strategic uncertainty," American Political Science Review, 79, 62-78.

Peña, J., G. NÖldeke, And L. Lehmann (2015): "Evolutionary dynamics of collective action in spatially structured populations," Journal of Theoretical Biology, 382, 122-136.

PeñA, J., L. Lehmann, And G. NÖldeke (2014): "Gains from switching and evolutionary stability in multi-player matrix games," Journal of Theoretical Biology, 346, 23-33. 